\title{
Are low-intensity CBT interventions effective and meaningful for the Latino community in the UK?
}

Jose E. Lopez (a), Melinda Rees (a), Maria Castro (b)

a) iCope - Islington Psychological Therapies and Wellbeing Service, London, UK;

b) Clinical Psychology Doctorate Programme, School of Psychology, University of East London, London, UK

In the UK, low-intensity cognitive-behavioural therapy (LICBT) is offered as cost-effective intervention for anxiety and depression in primary care. Whilst research with Latino migrants in the USA highlights the suitability of CBT interventions with this group, these findings may not be generalisable to the UK, due to different socio-political circumstances. This mixed-method study explores the effectiveness and meaning of an LICBT group-intervention for Latino migrants in London. Eight participants attended a fourweek workshop on anxiety management in Spanish, and pre- and post-intervention scores in CORE-OM, PHQ-9 and GAD-7 were compared. Additionally, focus groups about the intervention were thematically analysed.

Although not statistically significant, a trend towards decreasing anxiety levels was identified $(p=0.06)$. Three of the four themes generated from participants' analysis of their experience are summarised and discussed. These include positive aspects of the intervention and its limitations. Whilst valuing LICBT interventions, participants considered further input necessary. Potential clinical implications as well as recommendations for further research are discussed.

Keywords: low intensity CBT; Latinos; psychotherapy; culture

\section{Introduction}

Cultural factors have been increasingly recognised in mental health research and practice (Sewell, 2009) but have had little impact on the development of psychological theory and interventions (Patel, 1999). Consequently, making available a multicultural approach, sensitive to cultural nuances, has clashed with an evidence-based paradigm derived from restrictive research, which often fails to include cultural variables (Morales \& Norcross, 2010). Despite arguments for and against culturally adapting psychotherapies (La Roche \& Christopher, 2009; Sue, 2001), Griner and Smith's (2006), meta-analytic review emphasises the benefits of cultural adaptations, particularly when targeted to a specific ethnic group and conducted in their mother tongue. Equally, Morales and Norcross (2010) state the importance of the therapist's competence in the cultural and linguistic aspects of the clients and their experience in integrating these variables in a congruent manner.

In current UK healthcare guidelines (NICE, 2004, 2009, 2011), cognitive behavioural therapy (CBT) is the treatment of choice for a range of anxiety and mood disorders.

Consequently, CBT has become the main therapeutic modality offered within Improving Access to Psychological Therapies (IAPT), a governmental initiative aimed at delivering evidence-based interventions in primary care (Layard, 2006). Adopting a stepped care approach, different levels of therapeutic input aim to suit different levels of severity, from guided self-help (i.e., low-intensity) to formulation-based (high-intensity) CBT interventions (Clark et al., 2009). The literature describing the strengths and limitations of CBT when used with people from a non-Western background favours its effectiveness across cultures (e.g., Hays, 1995). Although some evidence exists of the benefits of lowintensity CBT (LICBT) interventions, limited research has focused on its effectiveness with people from different ethnic minorities (Rathod \& Kingdon, 2009), rendering the value of this initiative for these groups as requiring further research. Official UK estimates describe the Latino community as 
a sizeable collective (Linneker \& Mclllwaine, 2011) despite marked discrepancy between figures due to the nonexistence of an official 'Latin American' ethnic category. They are a relatively recent community, arriving in migratory waves since the 1970s, from countries experiencing political unrest, conflict or economic crises (Mcllwaine, 2007). Their make-up has changed considerably depending on their country of origin and the nature of the migratory wave, generating collectives of different social classes, economic status and educational background (Carlisle, 2006). Mcllwaine (2007) states that the vicissitudes the average low-income Latino migrant in London faces daily relate to an overall poor command of English and their migration status. These two commonly cited problems lie at the heart of their employment difficulties (i.e., lack of work or exploitation), poor housing, limited access to services and opportunities for socialising. Household incomes substantially below the UK average render them more vulnerable to the global financial crisis (Mcllwaine, Cock, \& Linneker, 2011). Among women, the threat of deportation, physical abuse and lack of childcare support or benefits combine to make them the target of further discrimination (Carlisle, 2006). Despite these problems, a large number of Latinos still migrate to the UK in the hope of new opportunities, enduring incredibly precarious situations (Mcllwaine, 2007). The process of acculturation, common among international migrants, can be a further source of distress, which makes coping with these vicissitudes even more difficult (Bhugra, 2004).

Literature on Latino culture is full of stereotypical values and traits that describe a homogeneous collective and obviates marked variation in social class, education, degree of acculturation or place of origin. Nonetheless, anthropological, organisational (Hofstede, 2001) and psychotherapy research (Ardila-Espinel, 1982) define widespread values, such as familism, the importance of spiritual/religious beliefs, fatalism, formalism or the expectation of 'desahogo' (relief) (Interian \& Díaz-Martínez, 2007; Organista \& Muñoz, 1996; TorresRivera, 2004). These values may influence Latinos' stance towards therapy and their relationship to the therapist, their problem and their ability to change. Reviews of research on the use of CBT with Latinos in the USA show relative effectiveness among low-income individuals with anxiety or depression (Miranda et al., 2005; Morales \& Norcross, 2010). However, the generalisation of these findings to the UK Latino community may be limited by aspects such as its different size, different countries of origin or its establishment in both countries (Organista, 2006). Other influential sociopolitical factors are the existence of a National Health Service in the UK but not in the USA and differences in immigration systems in both countries.

\section{Methods}

In light of the overall lack of research into the effectiveness of mainstream psychological interventions for people from different cultural backgrounds in the UK, this study aimed to ascertain whether standard group LICBT intervention delivered in Spanish to first generation Latino migrants is effective (i.e., reduces the level of anxiety experienced by the participants as indicated by standardised outcome measures) and meaningful (i.e., fits with the participants' understanding of distress, as discussed in focus group interviews), as well as its beneficial elements.

A mixed-method approach seemed the most suitable and comprehensive approach to ascertain the effectiveness of the interventions whilst also gathering participants' views on the intervention and elements contributing to its overall effect. The study consisted in a Triangulation design - Convergence model (Creswell \& Plano Clark, 2007) with the quantitative and qualitative strands given equal weight and implemented during the same timeframe, collected and analysed separately and then converged at interpretation stage.

A critical realist standpoint enabled awareness of the material-discursive-intrapsychic concomitants of experiences constructed as distress, conceptualising them as mediated by culture, language and politics (Bhaskar, 1989). 


\section{Research design}

The quantitative part of the research draws intrasubject comparisons between the scores of each participant on several questionnaires (see below), completed at three different stages (Figure 1):

(1) Waitlist/control phase: 4-week period between registration and the beginning of the intervention

(2) Intervention phase: 4-week period of intervention

(3) Follow-up phase: 6 weeks post-intervention.

The qualitative part consisted of two focus groups, facilitated one week after the end of the intervention, for those participants who completed the intervention. This was complemented with the analysis of the most important elements of the intervention, as stated by participants at every session.

\section{Intervention}

A programme covering transdiagnostic aspects of anxiety and worry (i.e., physiological arousal, behavioural avoidance, cognitive biases) was compiled; the intervention was facilitated in Spanish.

Following similar programmes offered in IAPT services, the intervention was structured as four 90-minute stand-alone (to minimise drop-outs due to missed material) sessions:

(1) Introduction: physiology of anxiety and explanation of the CBT model

(2) Physiology: arousal reduction and relaxation techniques

(3) Behaviour: graded exposure, behavioural activation and sleep hygiene

(4) Cognition: worry management and thought challenging techniques.

At the end of every session, homework tasks were suggested to encourage participants to practise the principles discussed.

The intervention comprised audio-visual and printed material based on various resources from four IAPT services and translated into Spanish. Further CBT self-help material in Spanish was accessed online and a relaxation script was recorded by the first author. Once developed, three qualified LICBT practitioners gave their views about the programme and modifications in length and focus were made accordingly.

The first author, a qualified LICBT practitioner, facilitated the intervention in Spanish. Two groups of up to 10 people were facilitated to cater for participants' availability. These took place in a Latino community organisation. A theory-practice balance was sought, encouraging participant discussion and practical exercises. Session handouts were facilitated to participants.

Eighteen participants were registered in the LICBT programme and allocated to a group. They were recruited via collaborating community organisations catering for Latinos across London to avoid selecting only individuals able to access mainstream services. An effort was made to recruit a diverse group of people in terms of age and gender.

\section{Inclusion and exclusion criteria}

Participants were London-dwelling first-generation adult Latino migrants (who were born and had spent at least 10 years of their childhood in their country of origin), experiencing or with past experience of anxiety, interested in learning coping techniques and able to attend all four sessions. Individuals expressing self-harm ideation, unwilling to participate in a group intervention or receiving therapy were excluded.

Approval was obtained from the University of East London's Ethics committee (n.) as academic institution sponsoring the study. 
Quantitative data collection and analysis

Validated Spanish versions of questionnaires routinely used in IAPT services were selected to provide data comparable to previous literature on the effectiveness of CBT interventions:

- Patient Health Questionnaire 9 (PHQ-9): this is a self-administered measure of 'depression', with good sensitivity and specificity (Kroenke, Spitzer, \& Williams, 2001). Diez-Quevedo, Rangil, Sánchez-Planell, Kroenke and Spitzer (2001) stated that the Spanish version is comparable to the original.

- Generalised Anxiety Disorder 7-item Scale (GAD-7). This is a self-administered screening tool for anxiety, with good reliability and validity (Spitzer, Kroenke, Williams, \& Löwe, 2006). García-Campayo et al. (2010) concluded that the Spanish version showed adequate sensitivity, specificity and satisfactory concurrent validity.

- Clinical Outcomes in Routine Evaluation-Outcome Measure (CORE-OM). This is a self-administered, 34-item scale, which addresses domains of subjective wellbeing, symptoms, functioning and risk. It shows satisfactory psychometric properties, especially in primary care services, (Evans, Mellor-Clark, Barkham, \& Mothersole, 2006) and the Spanish version has been used as psychotherapy outcome measure (Botella, 2006).

- Short Acculturation Scale for Hispanics (SASH). The SASH identifies Hispanics who are low or high in acculturation. Despite its documented psychometric characteristics (Marín, Sabogal, VanOss Marín, Otero-Sabogal, \& Pérez-Stable, 1987), in this study it failed to discriminate among participants.

- Demographic questionnaire. A form was compiled to collect participants' demographic data to be completed at registration stage.

Participants' scores during control and intervention phases were compared using the Wilcoxon Signed Rank Test due to the small sample size and non-assumption of normal distribution. In addition, Exact procedures were run, as these remain valid for very small sample sizes and sparse or skewed data (Narayanan \& Watts, 1996). Exact Kruskal Wallis test was used to ascertain potential demographic factors explaining the study's attrition.

\section{Qualitative data collection and analysis}

Focus-group interviews were conducted as they capitalise on group dynamics, offer a more 'natural' environment (similar to the intervention setting) and are a time-efficient alternative to individual interviews (Krueger \& Casey, 2009). These were moderated by the first author.

Two focus groups were run, attended by three and four people, respectively. They were six females and one male, aged between 23 and 76 years old. Whilst lower than the numbers suggested as optimal for a focus group (Morgan \& Scannell, 1998), two separate sessions were run (specific for participants of either group) to minimise an undue effect of mixing participants from different groups on the discussion. To enhance discussion, a week before the focus groups participants were given pointers to reflect on. The groups were semistructured, following a pre-defined agenda, but also exploring aspects highlighted by the participants.

Discussions were tape-recorded and transcribed verbatim. A thematic analysis was conducted on the original (i.e., non-translated) transcripts of the focus group interviews to identify themes in participants' discussion. Thematic analysis was chosen due to its compatibility with a Critical Realist epistemology, allowing exploration of individuals' meaning-making of their experience and the influence of the broader social context on these meanings (Braun \& Clarke, 2006). Data were worked through following the steps described in Braun and Clarke's paper, namely: familiarisation with the data; generating initial codes; searching for themes; reviewing themes; defining and naming themes; producing the report. 


\section{Results}

Quantitative analysis

Outcome data

1. Control phase. The Wilcoxon Signed-ranks test performed with the participants' outcome measure scores showed subtle but non-significant reductions between control and preintervention stages:

- CORE-OM scores decreased by 1.3 standard deviations $(Z=-1.26, p=.23)$

- PHQ-9 scores decreased by 1.2 standard deviations $(Z=-1.19, p=.31)$

- GAD-7 scores decreased by 0.4 standard deviations $(Z=-.39, p=.78)$

2. Intervention phase. The Wilcoxon Signed-ranks test between scores at the beginning and end of the intervention showed a more noticeable drop in all three measures between first session and focus group than between registration and first session, especially in CORE-OM and GAD-7, which virtually reach the statistical significance threshold:

- CORE-OM scores decreased by nearly 2 standard deviations $(Z=-1.99, p=.06)$

- PHQ-9 scores decreased by 1.5 standard deviations $(Z=-1.53, p=.17)$

- GAD-7 scores decreased by 3 standard deviations $(Z=-3.03, p=.06)$.

3. Follow-up. Due to the poor completion rate of follow-up questionnaires (i.e., four participants' questionnaires were fully completed) these results cannot be reported.

Attrition analysis

Out of 15 participants who started, 10 completed the intervention (Figure 1). Whilst three participants put forward unexpected changes in circumstances (e.g., work or family commitments), reasons for attrition could not be ascertained in all cases due to ethical limitations.

No factors differentiate participants who completed the intervention from those who did not start it or dropped out. Table 2 shows the results of the Kruskal-Wallis test performed; neither age nor educational attainment, explain the participants' attendance.

Acculturation (as measured by the SASH and years of residence in the UK) is not associated with attendance. Equally, severity of distress at registration fails to explain their engagement.

\section{Qualitative analysis}

Thematic structure

A thematic analysis of the focus-group discussions yielded 4 themes and 13 subthemes. Table 3 comprises the final thematic structure. An analysis of valuable aspects of the nonspecific factors. Further questioning led to a discussion of aspects to be improved. Additionally, throughout the discussions, participants referred to wider contextual issues common to the Latino community in the UK and further support needed.1

1. Specific LICBT elements seen as useful. Participants stressed the importance of the techniques explained to reduce their excessive physical arousal and worry, describing some positive outcomes (e.g., better sleep and feeling calmer) and feeling 'empowered' by their new learning:

The breathing techniques were phenomenal ... (Participant 2, group A)

The topic of worry was helpful ... (Participant 3, group B)

Now I understand it's bad to avoid. (Participant 1, group B) 
This helps because you must be your own psychologist. (Participant 2, group B)

Participants emphasised the importance of gaining a professional understanding of anxiety, deeming the CBT model of anxiety 'the most important aspect'. Lacking this knowledge was described as contributing to anxiety. The 'accessible and comprehensive' material and the open dialogue format helped gain this understanding:

The most important [aspect] is to know what anxiety is at a professional level. (Participant 2, group B)

Had I known this earlier, [my crisis] wouldn't have been such ... (Participant 2, group B)

We had the chance to participate and discuss. (Participant 1, group A)

Questionnaire completion was perceived as a means to ascertain the severity of one's problem and how this improved:

Completing [questionnaires] is good to know how you are, where you started... (Participant 3, group B)

Participants stated the importance of practising regularly their newly learnt techniques. Suggested intersession tasks were deemed 'important' in their own right and as a means of expressing gratitude:

Now we have to continue to practise, to read. (Participant 1, group A)

Homework tasks are very important. (Participant 2, group A)

There you can see the gratitude for what he [facilitator] has done. (Participant 1 , group A)

2. Generic aspects of the intervention. Some facilitator characteristics described as conducive to participants' engagement were his kindness and professional manner, especially important during the first contact. Equally, participants emphasised them importance of a shared culture:

You are kind. ... We don't feel intimidated here. (Participant 2, group A)

I appreciate your professional qualities. (Participant 2, group A)

I thought it was a warm invite. (Participant 4, group B)

If you were English, you wouldn't understand our ways. (Participant 2, group A)

Participants praised receiving an intervention in their mother tongue and described their struggle to express their difficulties in English. They also voiced concerns about an increasing restriction of interpreting support:

Latinos here can't express themselves. (Participant 1, group B)

Having an interpreter with these cuts ... is not going to happen. (Participant 4, group B) 
Few people have access to professional information in one's language ... (Participant 1 , group A)

Participants described the group intervention as source of relief resulting from sharing experiences, emphasising its normalising and liberating effect and the opportunity for exposure it offers:

It works as a group to be able to share experiences. (Participant 1, group B)

Here I don't feel I am the only one with my problem. (Participant 2, group A)

It's a swimming pool were you push that person, giving them techniques to socialize.

(Participant 1, group B)

Peer-support was an essential ingredient, leading to widening people's networks beyond the session:

And you start to create a network with people. (Participant 4, group B)

It's you and not God that is doing the miracle. (Participant 1, group A)

Among the negative aspects, apprehension about a group intervention led some people to consider alternative support:

I thought 'everyone will comment outside on what I say'. (Participant 2, group A)

I wasn't prepared to share with others personal stuff. (Participant 3, group A)

3. Limitations of the intervention. Despite the positive comments above, participants' stressed the 'basic' nature and limited impact of the intervention. Suggestions for improvement ranged from allotting longer time to exploring the contents covered to group psychotherapy:

You must tell us when the next one is. l'll be here for sure. We need it. (Participant 3, group B)

We don't stop here. I'm now feeling better but need to continue. (Participant 2, group A)

Then you should say to the people: 'what do you think about confronting the issues, touching the wounds? (Participant 2, group A)

Several practical changes were suggested to the format of the intervention and audience make-up, which will not be covered here. All themes described above were endorsed by more than one participant in different discussion groups (as part of unrelated conversations), which was an important criterion for the selection of themes.

\section{Discussion}

This is the first study on psychological therapies carried out with Latinos living in the UK. It shows modest (non-statistically significant) effects of a group LICBT intervention with Spanish-speaking Latino migrants in the UK. This is in contrast with a more positive qualitative feedback during discussion with the participants, which emphasises a number of CBT-specific and other generic factors as therapeutic. 
Results show improving scores during the intervention phase, reaching levels close to those conventionally accepted as statistically significant, which are not registered during the control phase. However, the small sample size does not allow comparisons other than those reported above, limiting our understanding of the impact of the intervention.

Equally, our attempts to measure clinical significance were thwarted by the low numbers of questionnaires collected during the follow-up period. No demographic characteristics were identified as potential explanation of participants' engagement, which could be explained by the small sample size. However, it could also indicate that other factors might have influenced it (e.g., motivation, competing commitments).

Psychometric limitations of the outcome measures used (non-exhaustive rating categories, double-barrelled questions) might partially explain the results obtained. Equally, their focus (i.e., excessively sympton-based, dichotomy between anxiety and depression) does not reflect the potential for comorbidity (Pilgrim \& Bentall, 1999). Furthermore, the SASH failed to discriminate between participants, which shows the insufficiency of language use as an indicator of an individual's acculturation.

\section{Meaning of the intervention}

The focus groups generated a largely positive critique of the intervention as participants elaborated on what they deemed positive aspects of the intervention.

\section{Specific LICBT elements considered useful}

Participants' prioritisation of anxiety management skills over conceptual elements concurs with literature that describes CBT's emphasis on education as suitable to Latinos, who view life as an instructive experience (Comas-Díaz, 2006). The immediate benefits and ease of use of the techniques discussed by some participants -and reported in previous literature (Hays, 1995)- might underlie the importance given to this element.

Conversely, people appreciated gaining an understanding of the inner workings of anxiety and worry. The CBT conceptualisation seemed to be readily understood and to provide a logical explanation of experiences that most participants could relate to. The physiological slant given to the explanation of anxiety may have made this model more suited to a culture with high prevalence of somatisation (Escobar, 1995). Participants' praise of professional and scientific knowledge reflects their endorsement of the medical model as explicative of distress, as described by Peluso and Blay (2004).

Whilst acknowledging the importance of intersession tasks, participants failed mostly to regularly complete these. This discrepancy is apparent in other studies. Aguilera et al. (2010) describe that participants in previous intakes of the intervention emphasised the importance of these tasks to new members in the group, despite their own irregular completion rate. This might reflect their position in relation to the facilitator (respect for his authority) or the approach (reverence of scientific knowledge). Sessional completion of the standardised questionnaires was deemed a useful element of the intervention. Some saw it as a helpful element as it provided an objective measure of the way they felt, allowing them to monitor their progress. This is an element also highlighted by researchers in similar group interventions with Latinos (e.g., Aguilera et al., 2010).

Generic aspects of the intervention

Participants' emphasis on the facilitator's role illustrates the importance of the relationship with the therapist in the CBT literature (Hardy, Cahill, \& Barkham, 2009). 
However, it contrasts with similar studies on depression, which state that client evaluation of the therapist was weaker than other factors as predictor of positive outcomes in group CBT interventions with non-Latino participants (Oei \& Shuttlewood, 1997). Their stress on sharing culture with the therapist concurs with research on the advantages of ethnic matching in therapy (Griner \& Smith, 2006). However, participants' praise of the facilitator's performance may actually reflect positive features of the model (i.e., participants praised the facilitator's clear explanations instead of the clarity of the model). The importance of personal relations and the affectionate nature of Latinos (López \& Katz, 2001) may have also contributed to stressing this element.

Participants' appreciation of an intervention delivered in Spanish concurs with previous literature (Griner \& Smith, 2006; Torres-Rivera, 2004) emphasising the enhancing effects of using one's mother tongue in enabling better engagement and outcomes twice as effective as interventions in non-native language.

Participants' comments reflected Yalom's (1995) factors of 'imparting information' and 'universality', identified by Bieling, McCabe and Anthony (2006) as active ingredients of CBT group interventions. The emphasis on the former concords with Oei and Shuttlewood's (1997) study, which showed that specific CBT factors were more associated with reduction in depression severity than non-specific ones. Similarly, Aguilera et al. (2010) emphasise the role of mood-management methods as having a longer impact than group support. Intervention as limited/scope for improvement.

Participants' opinion of the intervention as limited in its scope and 'basic' concurs with previous qualitative studies on LICBT interventions (Khan, Bower, \& Rogers, 2007; Macdonald, Mead, Bower, Richards, \& Lovell, 2007), which depict this level of input as helpful support whilst awaiting more intensive interventions. A possible reason for such a judgement might be its focus on the individual, leaving aside relational complexities, as suggested by Comas-Díaz (2006).

The varying suggestions for further intervention made by the participants indicate the different expectations with which they approach help offered to them, which in turn shape their experience (Macdonald et al., 2007). Some participants' difficulty describing the support expected (e.g., using metaphorical language) may denote an excessive reliance on professional judgement, consistent with the high power orientation by which Hofstede (2001) describes the Latino culture.

\section{Convergence of quantitative and qualitative analyses}

The discrepancy between both parts of this study, also apparent in Aguilera's et al. (2010) work, can be interpreted in two ways:

(1) Prioritising the qualitative element - several factors may have affected the statistical comparisons. Firstly, the small sample size may have yielded unduly insignificant differences. The floor effect generated by some participants' low pre-intervention scores might have also affected comparisons. Finally, using symptom-focused standardised measures may have prevented from monitoring relevant outcomes.

(2) Prioritising the quantitative element, due to factors that might contribute to minimising criticism, such as fear of service withdrawal following negative feedback. Equally, stereotypical 'Latino' features (e.g., kindness and high deference to power) might also accentuate this lack of criticism. Finally, an attempt to be accepted (in order to fit in), common among marginalised minorities (Berry, 2001), could have also influenced the results. 
These discrepant results indicate a need for further research in a field lacking evidence base.

\section{Limitations}

This study was conducted as a first step towards gathering a culture-specific evidence base, due to the lack of previous research with Spanish-speaking Latinos in the UK.

However, attempting to explore the needs of this community based on a small sample is inherently an overgeneralisation.

\section{Sample}

A $67 \%$ completion rate compares favourably with similar studies with Latinos in the USA (e.g., Organista \& Muñoz, 1996). Of these, $70 \%$ attended the focus groups, showing active involvement, with those with experience of anxiety difficulties being more participative. However, insufficient follow-up measures limit our findings to the immediate effects of the intervention.

\section{Statistical analysis}

Despite using appropriate statistical tests (Wilcoxon Signed Ranks) with exact procedures to maximise their validity, the low numbers attending the groups only allow modest conclusions to be drawn on the effectiveness of this therapy.

\section{The researcher's double role}

The researcher's double role as facilitator of the intervention and the focus groups may have influenced participants' evaluation of the intervention, generating unduly positive qualitative feedback. Nonetheless, this bears strong similarities with routine practice in IAPT services, where therapists themselves review therapy outcomes with their clients at the end of treatment, thus lending ecological validity to this study.

\section{Clinical implications}

\section{For service users}

This study indicates that group CBT-informed self-help interventions, whilst not showing compelling evidence of effectiveness, are well received by participants, who voice the need for suitable interventions in the community.

\section{For practitioners}

Interventions should also strike a balance between information-giving and providing space for relief, and participants' contribution to the group (i.e., peer support). Extra emphasis might be required when working with this community to enhance completion of homework tasks and hence therapeutic effectiveness.

\section{For policy-makers}

Therapeutic interventions should be facilitated in the person's mother tongue by a therapist familiar with their culture. At a time of budget cuts in health services, language matching might be seen as an overly luxurious option, which is, nonetheless, necessary to cater for minority communities' needs. Group interventions like the one evaluated in this study, offered by native speakers or, in their absence, via interpreters, might be more affordable options. 


\section{Further research}

To confirm these preliminary findings, further research with a larger sample would allow more robust statistics. Further work should also focus on individual low-intensity interventions offered to Latinos and comparing its outcomes to other ethnic minorities.

Finally, the lack of consistent evidence supporting LICBT interventions indicates that other ways of working with the Latino community should be explored. In this study the participants' willingness to participate in research was apparent. However, participation was affected by the competing demands rife among socioeconomically deprived communities (e.g., unreliable working patterns, childcare).

\section{Notes on contributors}

Dr Jose E. Lopez completed his doctorate in Clinical Psychology at the University of East London. He currently works as a Clinical Psychologist in a primary care psychology service and coordinates the mental health provision of a London-based Latin American community organisation.

Dr Melinda Rees is a Team Manager in iCOPE - the Increasing Access to Psychological Therapies (IAPT) Service in Islington, London. She completed her DClin Psy at the University of East London 11 years ago and has also studied CBT at Postgraduate level at Oxford. She has a particular clinical interest in trauma and has worked in Primary Care across many cultural groups.

Dr Maria Castro is Senior Lecturer and Academic Tutor for the Professional Doctorate in Clinical Psychology at the University of East London. Previously, she was an NHS Clinical Psychologist in Older People's Services. Maria's core interests are in ethical, creative and collaborative praxis, particularly with people and communities largely marginalised.

\section{References}

Aguilera, A., Garza, M., \& Muñoz, R. (2010). Group cognitive-behavioral therapy for depression in Spanish: Culture-sensitive manualized treatment in practice. Journal of Clinical Psychology, 66(8), 857-867. doi:10.1002/jclp.20706

Ardila-Espinel, N. (1982). Criterios y valores de la cultura anglo-saxo-americana y de la cultura latina: sus implicaciones para la psicología transcultural [Criteria and values of the anglo-saxoamerican and Latino cultures: Implications for transcultural psychology]. Revista Latinoamericana de Psicología, 14(1), 63-79.

Berry, J. W. (2001). A psychology of immigration. Journal of Social Issues, 57(3), 615-631. doi:10.1111/0022-4537.00231

Bhaskar, R. (1989). Reclaiming reality: A critical introduction to contemporary philosophy. London: Verso.

Bhugra, D. (2004). Migration, distress and cultural identity. British Medical Bulletin, 69(1), 129-141. doi:10.1093/bmb/ldh007

Bieling, P., McCabe, R., \& Anthony, M. (2006). Cognitive-Behavioral therapy in groups. New York: Guilford.

Botella, L. (2006). Routine evaluation in a psychotherapy service: The use of CORE system data. European Journal of Psychotherapy \& Counselling, 8(2), 235-241. doi:10.1080/13642530600714672

Braun, V., \& Clarke, V. (2006). Using thematic analysis in psychology. Qualitative Research in Psychology, 3(2), 77-101. doi:10.1191/1478088706qp063oa

Carlisle, F. (2006). Marginalisation and ideas of community among Latin American migrants to the UK. Gender \& Development, 14(2), 235-245. doi:10.1080/13552070600747230

Clark, D. M., Layard, R., Smithies, R., Richards, D. A., Suckling, R., \& Wright, B. (2009). Improving access to psychological therapy: Initial evaluation of two UK demonstration sites. 
Behaviour Research and Therapy, 47(11), 910-920. doi:10.1016/j.brat.2009.07.010

Comas-Díaz, L. (2006). Latino healing: The integration of ethnic psychology into psychotherapy. Psychotherapy: Theory, Research, Practice, Training, 43(4), 436-453. doi:10.1037/0033-3204.43.4.436

Creswell, J., \& Plano Clark, V. (2007). Designing and conducting mixed methods research. Thousand Oaks, CA: Sage.

Diez-Quevedo, C., Rangil, T., Sánchez-Planell, L., Kroenke, K., \& Spitzer, R. (2001). Validation and utility of the patient health questionnaire in diagnosing mental disorders in 1003 general hospital Spanish inpatients. Psychosomatic Medicine, 63(4), 679-686.

Escobar, J. (1995). Transcultural aspects of dissociative and somatoform disorders. Psychiatric Clinics of North America, 18(3), 555-569.

Evans, R., Mellor-Clark, J., Barkham, M., \& Mothersole, G. (2006). Developing the resources and management support for routine evaluation in counselling and psychological therapy service provision: Reflections on a decade of CORE development. European Journal of Psychotherapy \& Counselling, 8(2), 141-161. doi:10.1080/13642530600712494

García-Campayo, J., Zamorano, E., Ruiz, M. P., Pérez-Páramo, M., López-Gómez, V., Freire, O.,Rejas, J. (2010). Cultural adaptation into Spanish of the generalized anxiety disorder-7 (GAD-7) scale as a screening tool. Health and Quality of Life Outcomes, 8(8), 111.

Griner, D., \& Smith, T. B. (2006). Culturally adapted mental health intervention: A metaanalytic review. Psychotherapy: Theory, Research, Practice, Training, 43(4), 531-548. doi:10.1037/0033-3204.43.4.531

Hardy, G., Cahill, J., \& Barkham, M. (2009). Active ingredients of the therapeutic relationship that promote client change. A research perspective. In P. Gilbert \& R. Leahey (Eds.), The therapeutic relationship in the cognitive behavioral psychotherapies (pp. 24-42). Hove: Routledge.

Hays, P. A. (1995). Multicultural applications of cognitive-behavior therapy. Professional Psychology: Research and Practice, 26(3), 309-315. doi:10.1037/0735-7028.26.3.309

Hofstede, G. (2001). Culture's consequences: Comparing values, behaviours, institutions and organisations across nations (2nd ed.). Thousand Oaks, CA: Sage.

Interian, A., \& Díaz-Martínez, A. M. (2007). Considerations for culturally competent cognitive behavioral therapy for depression with Hispanic patients. Cognitive and Behavioral Practice, 14 (1), 84-97. doi:10.1016/j.cbpra.2006.01.006

Khan, N., Bower, P., \& Rogers, A. (2007). Guided self-help in primary care mental health: Metasynthesis of qualitative studies of patient experience. The British Journal of Psychiatry, 191(3), 206-211. doi:10.1192/bjp.bp.106.032011

Kroenke, K., Spitzer, R. L., \& Williams, J. B. W. (2001). The PHQ-9: Validity of a brief depression severity measure. Journal of General Internal Medicine, 16(9), 606-613. doi:10.1046/j.1525-1497.2001.016009606.x

Krueger, R., \& Casey, M. (2009). Focus groups: A practical guide for applied research (4th ed.). London: SAGE.

La Roche, M. C., \& Christopher, M. S. (2009). Changing paradigms from empirically supported treatment to evidence-based practice: A cultural perspective. Professional Psychology: Research and Practice, 40(4), 396-402. doi:10.1037/a0015240

Layard, R. C. (2006). The depression report: A new deal for depression and anxiety disorders. London: The Centre for Economic Performance's Mental Health Policy Group, London School of Economics.

Linneker, B., \& Mclllwaine, C. (2011). Estimating the Latin American population of London from official data sources. London: Queen Mary's University of London.

López, A., \& Katz, I. (2001). An introduction to Latinos in the United States. In A. López \& E. Carrillo (Eds.), The latino psychiatric patient (pp. 3-18). Washington, DC: American Psychiatric Publishing.

Macdonald, W., Mead, N., Bower, P., Richards, R., \& Lovell, K. (2007). A qualitative study of patients' perceptions of a 'minimal' psychological therapy. International Journal of Social Psychiatry, 53(1), 23-35. doi:10.1177/0020764006066841 
Marín, G., Sabogal, F., VanOss Marín, B., Otero-Sabogal, F., \& Pérez-Stable, E. (1987). Development of a short acculturation scale for Hispanics. Hispanic Journal of Behavioral Sciences, 9(2), 183-205. doi:10.1177/07399863870092005

Mcllwaine, C. (2007). Living in Latin London: How Latin American migrants survive in the city. Institute for the Study of the Americas. Retrieved November 26, 2010, from http://americas.sas.ac.uk/newsletter/docs/LivinglnLatinLondon.pdf

Mcllwaine, C., Cock, J. C., \& Linneker, B. (2011). No longer invisible: The Latin American community in London. London: Queen Mary's University.

Miranda, J., Bernal, G., Lau, A., Kohn, L., Hwang, W, \& Lafromboise, T. (2005). State of the science on psychosocial interventions for ethnic minorities. Annual Review of Clinical Psychology, 1(1), 113-142. doi:10.1146/annurev.clinpsy.1.102803.143822

Morales, E., \& Norcross, J. (2010). Evidence-based practices with ethnic minorities: Strange bedfellows no more. Journal of Clinical Psychology, 66(8), 821-829. doi:10.1002/jclp.20712

Morgan, D. L., \& Scannell, A. (1998). Planning focus groups. London: Sage.

Narayanan, A., \& Watts, D. (1996). Exact methods in the NPAR1WAY procedure. UCLA Academic Technology Services. Retrieved March 16, 2012, from, http://www.ats.ucla.edu/stat/sas/library/exact.pdf

National Institute for Health and Clinical Excellence (NICE). (2004). Anxiety: Management of anxiety (panic disorder, with or without agoraphobia, and generalised anxiety disorder) in adults in primary, secondary and community care. London: Author.

National Institute for Health and Clinical Excellence (NICE). (2009). Depression: The treatment and management of depression in adults (update). London: Author.

National Institute for Health and Clinical Excellence (NICE). (2011). Guidelines for the treatment and management of post-traumatic stress disorder (PTSD) (CG26). London: Author.

Oei, T. P. S., \& Shuttlewood, G. J. (1997). Comparison of specific and nonspecific factors in a group cognitive therapy for depression. Journal of Behavior Therapy and Experimental Psychiatry, 28(3), 221-231. doi:10.1016/S0005-7916(97)00019-0

Organista, K. (2006). Cognitive-behavioral therapy with Latinos and Latinas. In P. Hays, \& G. Iwamasa (Eds.), Culturally responsive cognitive-behavioral therapy: Assessment, practice, and supervision (pp. 73-96). Washington, DC: American Psychological Association.

Organista, K. C., \& Muñoz, R. F. (1996). Cognitive behavioral therapy with Latinos. Cognitive and Behavioral Practice, 3(2), 255-270. doi:10.1016/S1077-7229(96)80017-4

Patel, N. (1999). Getting the evidence: Ethical guidelines for mental health research involving issues of 'race', ethnicity and culture. London: MIND.

Peluso, E., \& Blay, S. L. (2004). Community perception of mental disorders: A systematic review of Latin American and Caribbean studies. Social Psychiatry and Psychiatric Epidemiology, 39 (12), 955-961. doi:10.1007/s00127-004-0820-y

Rathod, S., \& Kingdon, D. (2009). Cognitive behaviour therapy across cultures. Psychiatry, 8(9), 370-371. doi:10.1016/j.mppsy.2009.06.011

Sewell, H. (2009). Working with ethnicity, race and culture in mental health. London: Jessica Kingsley.

Spitzer, R., Kroenke, K., Williams, J., \& Löwe, B. (2006). A brief measure for assessing generalized anxiety disorder: The GAD-7. Archives of Internal Medicine, 166(10), 10921097. doi:10.1001/archinte.166.10.1092

Sue, S. (2001). In defense of cultural competency in psychotherapy and treatment. American Psychologist, 42(1), 37-45. doi:10.1037/0003-066X.42.1.37

Torres-Rivera, E. (2004). Psychoeducational and counseling groups with Latinos. In J. L. DeLucia-Waack (Ed.), Handbook of group counseling and psychotherapy (pp. 213-223). London: Sage.

Yalom, I. (1995). The Theory and practice of group psychotherapy (4th ed.). New York: Basic Books. 